\title{
Synthesis of molecularly imprinted polymers of vanillic acid and extraction of vanillic acid from spiked blood serum
}

\author{
Showkat Ahmad Bhawani ${ }^{*}$, Salma Bakhtiar ${ }^{1}$, Rachel Roland ${ }^{1}$, Syed Rizwan Shafqat ${ }^{1}$, Mohamad Nasir Mohamad Ibrahim² \\ ${ }^{1}$ Faculty of Resource Science and Technology, Universiti Malaysia Sarawak (UNIMAS), Sarawak, Kota Samarahan 94300, Malaysia \\ ${ }^{2}$ School of Chemical Sciences, Universiti Sains Malaysia, Pulau Pinang 11800, Malaysia
}

\author{
ARTICLE INFO \\ Received on: 23/08/2019 \\ Accepted on: 29/01/2020 \\ Available online: 04/04/2020 \\ Key words: \\ Vanillic acid, molecular \\ imprinted polymer, \\ precipitation polymerization, \\ acrylic acid, ethylene glycol \\ dimethacrylate.
}

\begin{abstract}
Vanillic acid (4-hydroxy-3-methoxybenzoic acid) is a phenolic acid found in many plant extracts. It is used as a flavoring and scent agent and produces a pleasant, creamy odor. It is widely used in many applications for therapeutic purposes to flavoring agent. Molecularly imprinted polymers of vanillic acid were synthesized by precipitation polymerization with a noncovalent approach for the extraction from blood serum. Three different imprinted polymers have been synthesized with varying molar ratio of monomer. The synthesized polymer particles were characterized using Fourier-transform infrared spectroscopy and scanning electron microscopy. The extraction efficiency of highly selected imprinted polymer of vanillic acid from spiked blood serum was about $80 \%$.
\end{abstract}

\section{INTRODUCTION}

The molecular imprinting is a versatile and diverse methodology for the synthesis of antibody-like materials. The imprinted materials are widely used because of their high selectivity and sensitivity, long-term stability, chemical inertness, and insolubility in water and most organic solvents (Lei and Mosbach, 2008). The process of molecular imprinting involves the preparation of synthetic polymers with predetermined recognition cavities under the guidance of a template. The association of the template with polymer matrix involves various kind of interactions, namely, covalent bonding, hydrogen bonding, van der Waals forces, ionic interactions, metal coordination interactions, and hydrophobic effects. In general, two binding approaches are mainly used for the preparation of molecular imprinting polymers (MIPs) such as covalent and noncovalent. The noncovalent

"Corresponding Author

Showkat Ahmad Bhawani, Faculty of Resource Science and Technology, Universiti Malaysia Sarawak (UNIMAS), Sarawak, Kota Samarahan 94300, MalaysiaE-mail: sabhawani@gmail.com methodology is widely used in the preparation of MIPs due to flexibility in choosing the monomers and the possible template and also the ease of preparation. The binding forces involved in the noncovalent approach are hydrogen bonding, van der Waals forces, ionic interactions, and hydrophobic effects (Flores et al., 2000; Mayes and Mosbach, 1996).

Due to the many advantages of noncovalent approach, we have also adopted the same procedure for the preparation of MIPs. The most conventional method of polymerization involved is the formation of monolith of highly cross-linked polymers by free radical polymerization of the functional monomer with an excess of cross-linking agent in a porogenic solvent in the presence or absence of template. The monolith always requires grinding and sieving, which eventually provides MIP particles of irregular size and shape. To avoid these drawbacks of conventional monolithic MIPs, we attempted to produce microsphere particles by precipitation method. It has been reported that so many synthetic strategies have been adopted to produce microsphere polymer particles such as suspension (Sellergren, 1994), dispersion, and seeded polymerization (Hosoya et al., 1998; Yilmaz et al., 2002). The contribution of these methods is undoubtedly valuable but, due to the use of emulsions and stabilizers for the formation of 
microspheres in some case, may remain adsorbed on the surface of particles which, in turn, reduces the rebinding of the molecule to the material. Due to these disadvantages, we adopted a precipitation method devoid of emulsifiers or stabilizers.

Vanillic acid is the oxidized form of vanillin and is found in abundance in vanilla beans (Sinha et al., 2008; Sostaric et al., 2000) and Angelica sinensis (Huang and Sheu, 2006). It is also found as one of the metabolites in human beings after consumption of green tea infusions (Pietta et al., 1998). Vanillic acid exhibits a myriad of applications in various pharmacological activities, such as inhibiting snake venom activity (Dhananjaya et al., 2006; 2009), carcinogenesis (Vetrano et al., 2005), apoptosis (Huang et al., 2008a;2008b), and inflammation(Itoh et al., 2009). Due to its pleasant creamy odor, it is widely used in fragrances in various food items. Vanillic acid could be used as a safe and physiologically inert inducer for gene switches designed for future therapeutic applications. Therefore, the synthesis of MIPs of vanillic acid could open a variety of applications in various fields. These materials can be used for the delivery of vanillic acid into various systems and for its extraction from various biological samples. Therefore, we have proposed a method for the synthesis of microsphere MIPs of vanillic acid by precipitation without any use of emulsifier.

\section{MATERIALS AND METHODS}

\section{Materials and reagents}

Vanillic acid and 2,2'-azobis(isobutyronitrile) (AIBN) were obtained from R\&M Marketing Company located in Essex, UK. Acetonitrile was obtained from Avantor Performance Materials Incorporation located in Phillipsburg, New Jersey. Methacrylic acid (MAA) and ethylene glycol dimethacrylate (EGDMA) were obtained from Sigma-Aldrich Corporation located in St. Louis, Missouri. Acetone was obtained from $\mathrm{HmbG}$ Chemicals Company located in Hamburg, Germany, and the methanol and acetic acid were obtained from R\&M Marketing Company located in Essex, UK.

\section{Preparation of MIPs}

The polymers were synthesized by precipitation polymerization method. In the course of preparation, the following procedure was adopted: template $(0.5 \mathrm{mmol})$, MAA $(4 \mathrm{mmol})$, EGDMA (16 mmol), and AIBN $(0.30 \mathrm{mg})$ which were dissolved in round-bottom borosilicate one-necked flask. After this process, the whole solution mixture was sonicated for 10 minutes in a sonicator. The sonication was followed by the purging of nitrogen for 15 minutes in an ice bath. The purging by nitrogen is carried out to make the environment free from any interfering oxygen dissolved in the reaction mixture. After purging, the flask containing reaction mixture was kept in a temperature-controlled water bath. Initially, the temperature was raised ambient to $60^{\circ} \mathrm{C}$ for 3 hours and raised up to $80^{\circ} \mathrm{C}$ for the next 3 hours. After the polymerization process, the microspheres synthesized were washed with acetone thrice in an ultrasonic bath. The same procedure was adopted for the synthesis of other imprinted polymers [MIP2 (template: MAA: EGDMA, 0.5:6:20) and MIP3 (template: MAA: EGDMA, 0.5:8:20)] and nonimprinted polymer (NIP) (MAA: EGDMA, 4:20).
In the case of imprinted polymers, the template was removed from the polymer by centrifugation. In this process, the polymer was treated with methanol:acetic acid (80:20, v/v) and then centrifuged several times until the template was completely removed from the polymer. The removal of template was monitored by ultra violet-visible spectrophotometry.

\section{Fourier-transform infrared spectroscopy (FTIR) characterization and scanning electron microscopy (SEM) analysis}

The IR characterization provides information about the incorporation or binding of template in the polymer matrix by comparing shifts at various wavelengths.

SEM images provide information about the surface morphology (shape and size).

\section{Batch binding assay}

In the batch binding experiments, $0.5 \mathrm{~g}$ sample of different MIPs (MIP1, MIP2, and MIP3) and NIP is weighed and incubated in a beaker containing $1 \mathrm{mmol}$ of vanillic acid in $50 \mathrm{ml}$ of acetonitrile. The beaker is kept on a horizontal shaker for 240 minutes. The continuous shaking is carried out for 240 minutes. The samples from the beakers are collected at different time intervals $(30,60,90,120,150,180,210$, and 240 minutes). The collected samples were then analyzed by high-performance liquid chromatography (HPLC). The HPLC was performed using acetonitrile, water, and acetic acid (60:39.5:0.5) as an eluent and C18 column as a stationary phase. The flow rate of the sample was maintained at $1.0 \mathrm{ml} / \mathrm{min}$. The extraction percentage $(\%)$ of imprinted and NIP beads was calculated by the following equation:

$$
\text { Extraction efficiency }=\left(\frac{\mathrm{C}_{i}-\mathrm{C}_{f}}{\mathrm{C}_{i}}\right) \times 100
$$

where $\mathrm{C}_{i}$ and $\mathrm{C}_{f}$ are the initial and final concentrations of vanillic acid in the feed solution, respectively.

\section{Adsorption parameters}

The parameters affecting binding efficiency such as initial concentration, polymer dosage, and $\mathrm{pH}$ value were studied by batch binding experiments. All the parameters are given in Table 1. The same procedure was followed in all the cases as in rebinding assay.

\section{Selectivity study}

To evaluate the selectivity of vanillic acid MIP, the recognition experiments were conducted in which the selectivity of vanillic acid was compared with syringic acid as a structural analog of vanillic acid.

The selective adsorption experiments were carried out in the binary solution of vanillic acid and syringic acid. A series of binary solutions (10 ppm vanillic acid and $10 \mathrm{ppm}$ syringic acid) having the $\mathrm{pH}$ value of 7 were prepared. One set of binary solution was added with $400 \mathrm{mg}$ of MIP and another with $400 \mathrm{mg}$ of NIP. Both the sets were agitated on a shaker at $250 \mathrm{rpm}$ for an optimum 
Table 1. Different adsorption parameters.

\begin{tabular}{clcl}
\hline S. No. & Parameter & Variation in parameter & Constant parameter \\
\hline 1 & Different initial concentration & $10,15,20,25,30 \mathrm{ppm}$ & $\begin{array}{l}\text { Temperature: } 300 \mathrm{~K} \text {, agitation speed: } 250 \mathrm{rpm}, \text { contact } \\
\text { time: } 90 \text { minutes, adsorbent dose: } 400 \mathrm{mg}, \mathrm{pH}: 7\end{array}$ \\
2 & Different dosage & $100,200,300,400,500 \mathrm{mg}$ & $\begin{array}{l}\text { Temperature: } 300 \mathrm{~K} \text {, Agitation speed: } 250 \mathrm{rpm}, \text { Contact } \\
\text { time: } 90 \text { minutes, pH: } 7, \text { Concentration: } 20 \mathrm{ppm}\end{array}$ \\
& $\mathrm{pH}$ & $2,5,7,9,12$ & $\begin{array}{l}\text { Temperature: } 300 \mathrm{~K}, \text { agitation speed: } 250 \mathrm{rpm}, \\
\text { contact time: } 90 \mathrm{minutes} \text {, adsorbent dose: } 400 \mathrm{mg}, \text { and } \\
\text { concentration: } 20 \mathrm{ppm} .\end{array}$ \\
\hline
\end{tabular}

contact time of 90 minutes. Further analysis was carried out by reversed phase-HPLC.

The distribution and selectivity coefficient of vanillic acid as compared to syringic acid were calculated using the following equation:

$$
K_{\mathrm{d}}=\frac{\left[\mathrm{C}_{\underline{i}}-\mathrm{C}_{f}\right] \mathrm{V}}{\mathrm{M}}
$$

where

$K_{\mathrm{d}}=$ distribution coefficient,

$\mathrm{C}_{i}=$ initial concentration of binary solution,

$\mathrm{C}_{f}=$ final binary solution concentration,

$\mathrm{V}=$ volume of the binary solution, and

$\mathrm{M}(\mathrm{g})=$ the amount of MIP added.

The selectivity coefficient for vanillic acid in the presence of competitor syringic acid can be calculated from equilibrium binding efficiency according to the following equation:

$$
K=\frac{K_{\mathrm{d}}(\text { vanillic acid })}{K_{\mathrm{d}}(\text { syringic } \text { acid })}
$$

where

$\mathrm{K}=$ selectivity coefficient.

The relative selectivity coefficient $\mathrm{k}^{\prime}$ can be defined as follows:

$$
\mathrm{k}^{\prime}=\frac{\mathrm{K} \text { MIP }}{\mathrm{K} \text { NIP }}
$$

\section{Extraction of vanillic acid from spiked blood serum}

The extraction of syringic acid from blood serum was conducted by selected MIP with the highest binding capacity and the NIP as the control. About $9 \mathrm{ml}$ of drug-free fresh blood was collected, and then, it was allowed to clot for 30 minutes. The blood was centrifuged at 4,000 rpm for 10 minutes to extract the serum. About $3 \mathrm{ml}$ of blood serum was placed in a test tube and diluted with $30 \mathrm{ml}$ of distilled water. After that, $33 \mathrm{ml}$ of $50 \mathrm{ppm}$ stock solution of vanillic acid was spiked in the blood serum. This was followed by the addition of $0.5 \mathrm{~g}$ of MIP in a solution. The extraction procedure was conducted in the same way as followed in batch binding process.

\section{RESULTS AND DISCUSSION}

The synthesis of polymers was conducted using precipitation method. The binding of different components in the reaction mixture was carried out by noncovalent approach. In this research, different polymers have been synthesized by varying the molar ration of monomer and keeping other materials constant for all cases. The precipitation method of polymerization was used to produce microspheres. This is because the synthesis of microsphere imprinted polymers is very important for the production of uniform shape and size of particles. It was reported (Bai et al., 2006) that various preparation methods have been used for the preparation of polymer microspheres (Okubo and Nakagawa, 1994), such as the synthesis of polymer microspheres by dispersion and emulsion polymerization, where the surfactants in aqueous solution (Ferrick et al., 1989) and stabilizers in organic solution (Paine et al., 1990) are crucial to stabilize the polymer phase. Precipitation polymerization is capable for the formation of polymer microspheres with constant size and shape, without need of any added surfactant or stabilizer (Bai et al., 2005; Li and Stöver, 1993a; Romack et al., 1995; Sosnowski et al., 1996). This research was conducted in acetonitrile solvent and has successfully produced imprinted polymer microspheres.

\section{FTIR}

Figure 1 shows the FTIR spectra of MIPs and NIP. A broad peak of $\mathrm{OH}$ stretching is observed at 3,434-3,457 $\mathrm{cm}^{-1}$. This broad peak is due to the presence of $\mathrm{OH}$ group in the structure of AA. There is also a broad peak at 2,987-2,989 $\mathrm{cm}^{-1}$, which belongs to $\mathrm{CH}$ stretching. The $\mathrm{CH}$ stretching vibration is because of both the AA and EGDMA. A strong peak of $\mathrm{C}=\mathrm{O}$ of carboxylic acid stretching is present at 1,724.03-1,740.03 $\mathrm{cm}^{-1}$ and can be observed in both MIPs and NIP, corresponding to AA and EGDMA. The presence of strong stretching peak in the region of 1,145 (C-O) confirms the presence of EGDMA as crosslinking monomer (Pan et al., 2009). The strong peak at $1,727 \mathrm{~cm}^{-1}$ corresponds to the $\mathrm{CO}$ group of ester from that of EGDMA. A peak for the vibrational mode of $\mathrm{CH}$ binding and ring stretching can be seen in the region of 1,456-1,437 $\mathrm{cm}^{-1}$, which shows the presence of alkane group (from EGDMA) in both MIP and NIP. The antisymmetric and symmetric stretching bands of -O-R group of EGDMA appear at $1,159 \mathrm{~cm}^{-1}$ and $1,165 \mathrm{~cm}^{-1}$, respectively, in both MIP and NIP spectra which confirms the incorporation of EGDMA as a cross-linker (Bajpai and Jhariya, 2015; Gupta et al., 2016). The apparent shift in the absorption peak of $\mathrm{OH}$ stretching from $3,457 \mathrm{~cm}^{-1}$ in NIP to $3,458 \mathrm{~cm}^{-1}$ in MIP1 indicated the interaction of vanillic acid with the AA due to the possible hydrogen bonding. A small peak for $\mathrm{CH}$ binding can be seen at $1,040.85 \mathrm{~cm}^{-1}$. Some small peaks in the region of 844.77 and 918 $\mathrm{cm}^{-1}$ are observed for $\mathrm{CH}$ twisting and $\mathrm{CH}$ wagging, respectively. 


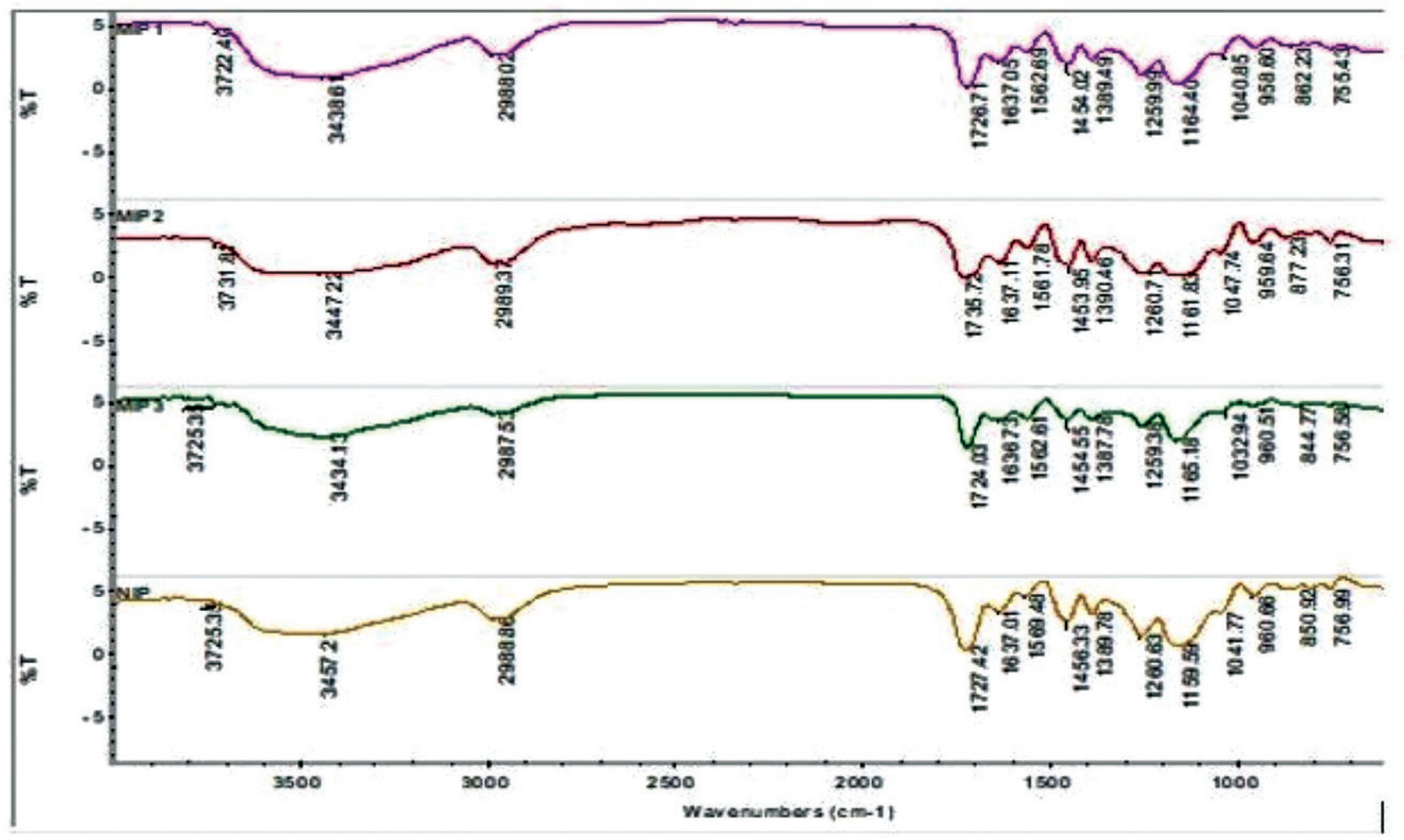

Figure 1. FTIR spectra of imprinted and non-imprinted polymers.

\section{SEM}

The surface morphology and particle size of the synthesized polymer can be explained by SEM analysis. Figure 2 shows that particles are highly uniform and spherical shape with sizes in micrometer. This is due to the polymer particles which were synthesized by precipitation polymerization method. A study reported that polymer synthesized by precipitation polymerization method produced uniform size of imprinted polymers (Tamayo et al., 2005). The size and shape of synthesized polymer are influenced by many factors including the type of polymerization method used, volume of solvent, temperature, and duration of synthesis. The highly uniform polymer microspheres can be produced in the presence of excess solvent in the synthesis of polymer particles (Roland and Bhawani, 2016).

\section{Batch binding study}

The rebinding efficiency of imprinted polymers was estimated by using batch binding study. Figure 3 shows that the MIP1 has the highest rebinding efficiency as compared to other MIPs in order MIP1>MIP2>MIP3. The highest rebinding efficiency or the time of equilibration was achieved at the time period of 180 minutes, and after that, it remained almost constant. The efficiency in the case of NIP was observed lowest with respect to all MIPs, and this may be due to the absence of complementary sites. While in the case of MIPs as reported by Yan and Row (2006), the imprinted polymer has permanent cavity for the template, and hence, an imprinted polymer will selectively bind with template molecule. Bergmann and Peppas (2008) stated that these cavities will not only sustain the shape of the desired template but also sustain the chemical functionalities from the complementary template. The low efficiency of MIPs (MIP2 and MIP3) may be due to the presence of scattered cavities in the polymer matrix (Pardeshi et al., 2014). The scattered binding sites have a low rebinding affinity for the template molecule.

\section{Effect of concentration}

The results from the experiments are shown in Figure 4 which shows that extraction efficiency/binding of vanillic acid molecules to the MIP increased from 10 to $20 \mathrm{ppm}$ until saturation of binding sites occurred. At $20 \mathrm{ppm}$, the adsorption process attains the highest rebinding efficiency after that the extraction of vanillic acid from the solution decreases slightly from 25 to $30 \mathrm{ppm}$ because of the saturation of MIP binding sites which means that vanillic acid molecules occupied all the active binding sites of MIP.

\section{Effect of MIP dosage}

The results from the MIP dosage study are depicted in Figure 5 which shows that the binding/extraction efficiency of vanillic acid MIP increases with an increase in MIP dosage up to $400 \mathrm{mg}$ after that the rebinding of vanillic acid from solution declines slightly. This can be explained by the fact that an increase in MIP dosage is directly proportional to the number of active binding sites available on the surface of MIPs (Tavengwa et al., 2016). Therefore, an optimum amount of MIP is required to achieve maximum rebinding efficiency. This means from the 


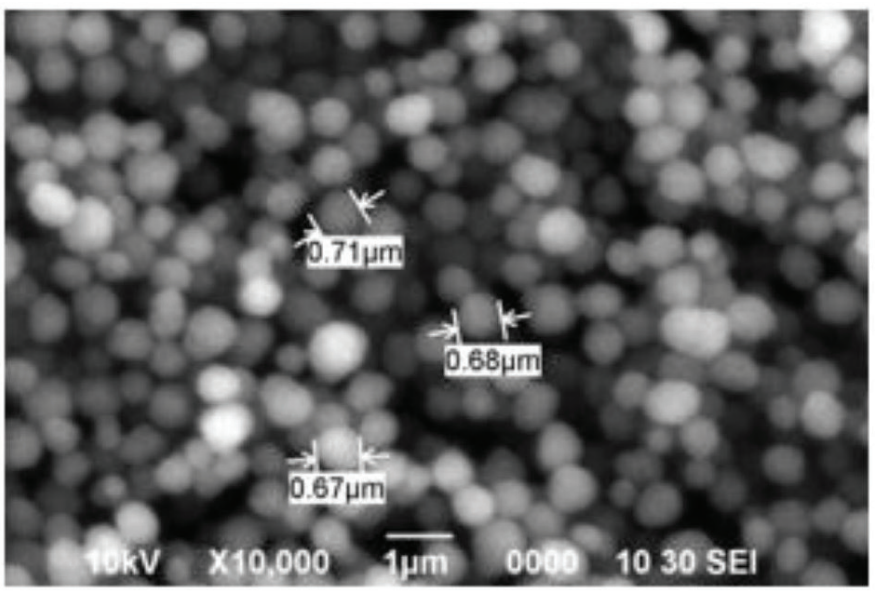

Figure 2. SEM of imprinted polymers.

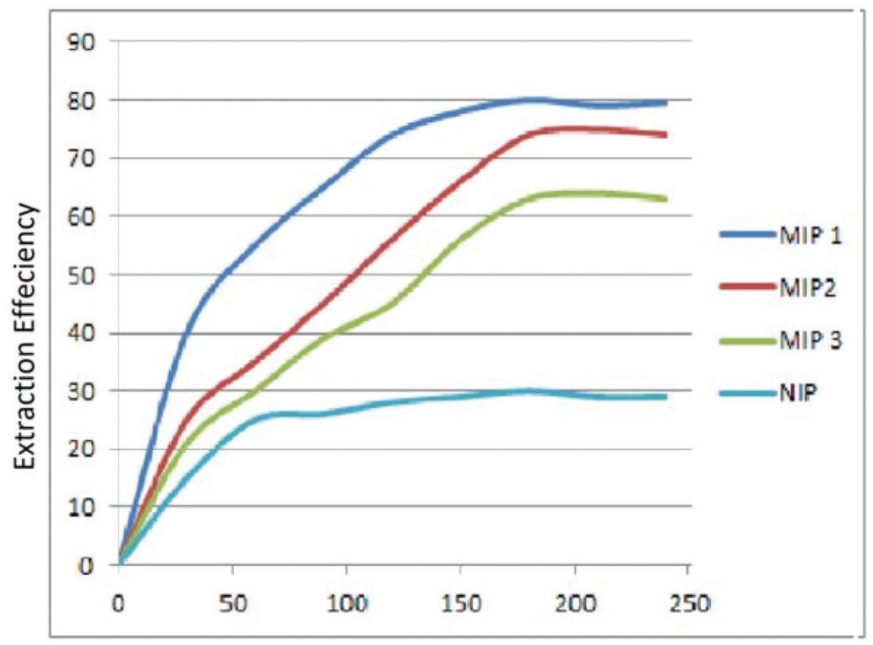

Time (Min.)

Figure 3. Re-binding efficiency of imprinted and non-imprinted polymers.

present study that $400 \mathrm{mg}$ is the optimum dosage to achieve maximum rebinding efficiency. The further increase in MIP dosage $(500 \mathrm{mg})$ has decreased the rebinding efficiency. This may be due to the formation of aggregate of polymer particles which might have decreased the access to the binding sites.

\section{Effect of pH}

The dependence of binding efficiency of vanillic acid MIPs on the $\mathrm{pH}$ solution which is one of the key factors in the process of adsorption was evaluated by varying the solution $\mathrm{pH}$ within the range of $2-12(2,5,7,9$, and 12). The solution $\mathrm{pH}$ not only brings about changes in the degree of the ionization but also affects the kinetics and subsequently the equilibrium of the process of adsorption (Yu et al., 2015). For pH study, the experiments were conducted by adding $400 \mathrm{mg}$ of vanillic acid MIP in $10 \mathrm{ml}$ of $20 \mathrm{ppm}$ vanillic acid solutions of different $\mathrm{pH}$ values $(2,5,7$, 9 , and 12). The $\mathrm{pH}$ of solutions was adjusted using 1 molar $\mathrm{HCl}$ and $\mathrm{NaOH}$ solutions. The outcomes of the study are shown in Figure 6 which confirms that the highest binding efficiency was

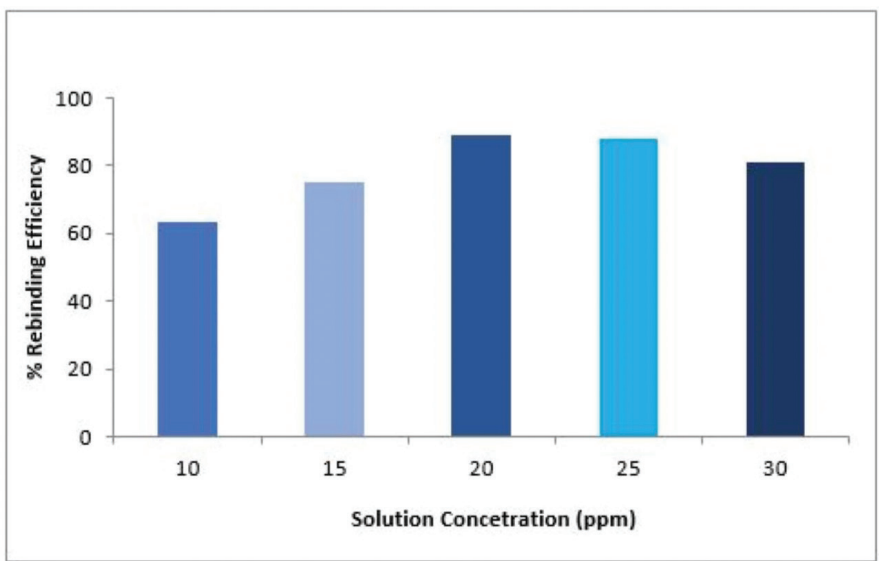

Figure 4. Effect of initial concentration of vanillic on rebinding efficiency of MIP.

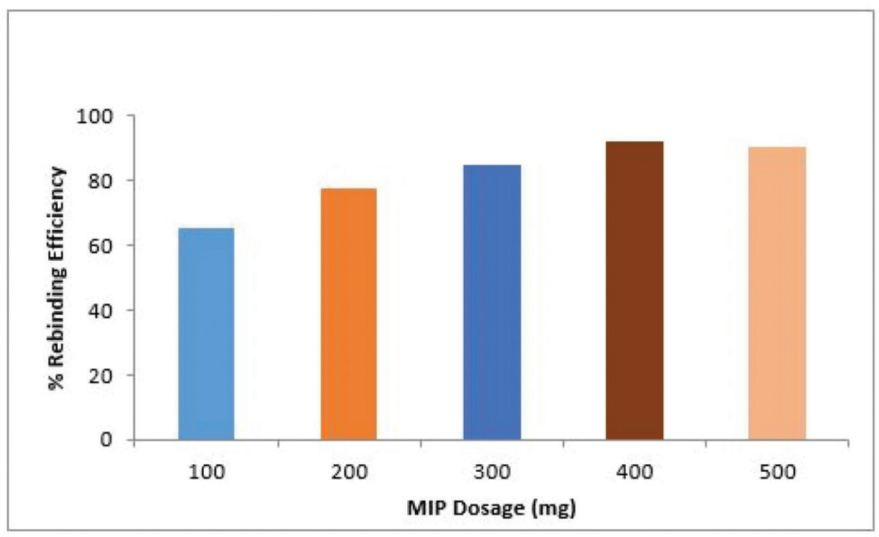

Figure 5. Effect of polymer dosage on rebinding efficiency.

achieved at $\mathrm{pH} 7$, and the binding efficiency of MIPs decreases at $\mathrm{pH}$ values lower or higher than 7 . This is because, at acidic or basic $\mathrm{pH}$, ionization of template (vanillic acid) occurs, which influences the electrostatic force of attraction and leads to repulsive forces between vanillic acid molecules and the active binding sites of MIP surface. These repulsive electrostatic forces overcome the binding efficiency of MIPs. Moreover, at $\mathrm{pH}$ higher or lower than 7, the higher concentration of $\mathrm{H}+$ and $\mathrm{OH}$-ions disturbs the $\mathrm{H}$-bonding between the template molecules and the active binding sites of MIP which is the main driving force of the process of adsorption (Dai et al., 2011).

\section{Selectivity of MIP}

The selectivity study of MIP deals with the extent/number of recognition/binding sites on the surface of MIP. The greater the recognition sites on MIP, the higher will be the selectivity of MIP and vice versa. Table 2 summarizes $K_{\mathrm{d}}$, k, and k' values of vanillic acid in comparison with the competitor syringic acid. The distribution ratio, selectivity, and relative selectivity for vanillic acid were found to be higher than syringic acid. This indicated that the MIP is more selective toward vanillic acid than syringic acid. It is also clear from the table that all the values obtained are higher for MIP as compared to NIP. This also provided information about the generation of binding sites selective toward the vanillic acid. 


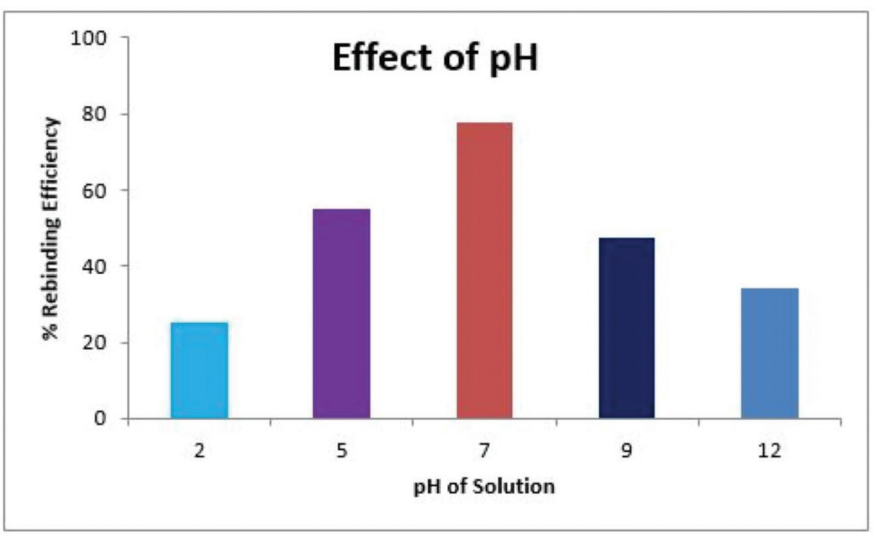

Figure 6. Effect of $\mathrm{pH}$ on rebinding efficiency of MIP.

Table 2. Selectivity of MIP.

\begin{tabular}{lcccc}
\hline Template & $\boldsymbol{K}_{\mathrm{d}}(\mathbf{M I P})$ & $\boldsymbol{K}_{\mathrm{d}}(\mathbf{N I P})$ & $\boldsymbol{k}$ sel & $\boldsymbol{k}^{\prime}$ \\
\hline Vanillic acid & 61 & 45 & 2.541 & 3.22 \\
Syringic acid & 24 & 57 & 0.789 & \\
\hline
\end{tabular}

\section{Application}

Vanillic acid is extensively used in variety of applications in various pharmacological activities such as inhibiting snake venom activity (Dhananjaya et al., 2006; 2009), carcinogenesis (Vetrano et al., 2005), apoptosis (Huang et al., 2008a; 2008b), and inflammation (Itoh et al., 2009). It has been found also as one of the metabolites in human beings after consumption of green tea infusions (Pietta et al., 1998). Therefore, it was important to synthesize MIPs for the extraction of vanillic acid from various biological samples. In this study, MIPI1 was used for the extraction of vanillic acid from blood serum, and it was found that about $80 \%$ of vanillic acid was successfully extracted from the spiked blood serum sample.

\section{CONCLUSION}

The precipitation of polymerization has been successfully applied to produce polymer particles with uniform shape and size. The morphology was characterized by SEM and was confirmed uniform shape and size of polymer particles. This could open a diverse application of the synthesized material such as for solid-phase extraction, column packing materials, and so on. In this study, a simple batch binding analysis was conducted for the removal of vanillic acid from spiked blood serum. A good amount $(80 \%)$ of vanillic acid was extracted from the blood serum. This indicated that the synthesized material is efficient for the purpose of extraction.

\section{DECLARATIONS}

\section{Competing interests}

The authors declared that they have no competing interests.

\section{ACKNOWLEDGMENTS}

This work was support by SGS UNIMAS under Grant F07(S168)/1243/2015(05) is highly appreciated.

\section{REFERENCES}

Bai F, Yang X, Li R, Huang B, Huang W. Monodisperse hydrophilic polymer microspheres having carboxylic acid groups prepared by distillation precipitation polymerization. Polym, 2006; 47:5775-84.

Bai F, Yang XL, Zhao YZ, Huang WQ. Synthesis of core-shell microspheres with active hydroxyl groups by two-stage precipitation polymerization. Polym Int, 2005; 54:168-74.

Bajpai SK, Jhariya S. Selective removal of Amikacin from simulated polluted water using molecularly imprinting polymer (MIP). J Macromol Sci, 2015; 52(11):901-11.

Bergmann NM, Peppas NA. Molecularly imprinted polymers with specific recognition for macromolecules and proteins. Prog Polym Sci, 2008; 33:271-88

Dai C, Geissen SU, Zhang YL, Zhang YJ, Zhou XF. Selective removal of diclofenac from contaminated water using molecularly imprinted polymer microspheres. Environ Pollut, 2011; 159(6):1660-6.

Dhananjaya BL, Nataraju A, Raghavendra Gowda CD, Sharath BK, D'Souza CJ. Vanillic acid as a novel specific inhibitor of snake venom 50-nucleotidase: a pharmacological tool in evaluating the role of the enzyme in snake envenomation. Biochemistry, 2009; 74:1315-9.

Dhananjaya BL, Nataraju A, Rajesh R, Raghavendra Gowda CD, Sharath BK, Vishwanath BS, D'Souza CJ. Anticoagulant effect of Naja naja venom 5 'Nucleotidase: demonstration through the use of novel specific inhibitor, vanillic acid. Toxicon, 2006; 48:411-21.

Ferrick MR, Murtagh J, Thomas JK. Synthesis and characterization of polystyrene latex particles. Macromolecules, 1989; 22:1515-7.

Flores A, Cunliffe D, Whitcombe MJ, Vulfson EN J. Imprinted polymers prepared by aqueous suspension polymerization. Appl Polym Sci, 2000; 77:1841.

Gupta MK, Bajpai J, Bajpai AK. Optimizing the release process and modelling of in vitro release data of cis-dichlorodiamminoplatinum (II) encapsulated into poly (2-hydroxyethyl methacrylate) nanocarriers. Mat Sci Eng, 2016; 58:852-62.

Hosoya K, Shirasu Y, Kimata K, Tanaka N. Molecularly imprinted chiral stationary phase prepared with racemic template. Anal Chem, 1998; 70:943.

Huang SM, Chuang HC, Wu CH, Yen GC. Cytoprotective effects of phenolic acids on methylglyoxal-induced apoptosis in Neuro-2A cells. Mol Nutr Food Res, 2008a; 52:940-9.

Huang SM, Hsu CL, Chuang HC, Shih PH, Wu CH, Yen GC. Inhibitory effect of vanillic acid on methylglyoxal-mediated glycation in apoptotic Neuro-2A cells. Neurotoxicology, 2008b; 29:1016-22.

Huang WY, Sheu SJ. Separation and identification of the organic acids in Angelicae Radix and Ligustici Rhizoma by HPLC and CE. Sep Sci, 2006; 29:2616-24.

Itoh A, Isoda K, Kondoh M, Kawase M, Kobayashi M, Tamesada M, Yagi K. Hepatoprotective effect of syringic acid and vanillic acid on concanavalin a-induced liver injury. Biol Pharm Bull, 2009; 32:1215-9.

Lei Y, Mosbach K. Molecular imprinting: synthetic materials as substitutes for biological antibodies and receptors. Chem Mater, 2008; 20:859-68.

Li K, Stöver HDH. Synthesis of monodisperse poly (divinylbenzene) microspheres. J Polym Chem A, 1993a; 31:3257-63.

Mayes AG, Mosbach K. Molecularly imprinted polymer beads: suspension polymerization using a liquid perfluorocarbon as the dispersing phase. Anal Chem, 1996; 68:3769.

Okubo M, Nakagawa T. Formation of multihollow structures in crosslinked composite polymer particles. Colloid Polym Sci, 1994; 272:530-5.

Paine AJ, Luymes W, McNulty J. Dispersion polymerization of styrene in polar solvents. 6 . Influence of reaction parameters on particle size and molecular weight in poly ( $\mathrm{N}$-vinylpyrrolidone)-stabilized reactions. Macromolecules, 1990; 23:3104-9.

Pan, G, Zu B, Guo X, Zhang Y, Li C, Zhang H. Preparation of molecularly imprinted polymer microspheres via reversible 
addition-fragmentation chain transfer precipitation polymerization. Polymer, 2009; 50(13):2819-25.

Pardeshi S, Dhodapkar R, Kumar A. Molecularly imprinted microspheres and nanoparticles prepared using precipitation polymerisation method for selective extraction of gallic acid from Emblica officinalis. Food Chem, 2014; 146:385-93.

Pietta PG, Simonetti P, Gardana C, Brusamolino A, Morazzoni $\mathrm{P}$, Bombardelli E. Catechin metabolites after intake of green tea infusions. Biofactors, 1998; 8:111-8.

Roland RM, Bhawani SA. Synthesis and characterization of molecular imprinting polymer microspheres of piperine: extraction of piperine from spiked urine. J. Anal Methods Chem, 2016; 1-6.

Romack TJ, Maury EE, DeSimone JM. Precipitation polymerization of acrylic acid in supercritical carbon dioxide. Macromolecules, 1995; 28:912-5.

Sellergren B. Direct drug determination by selective sample enrichment on an imprinted polymer. Anal Chem, 1994; 66:1578.

Sinha AK, Sharma UK, Sharma N. A comprehensive review on vanilla flavor: extraction, isolation and quantification of vanillin and others constituents. Int J Food Sci Nutr, 2008; 59:299-326.

Sosnowski S, Gadzionwski M, Slomkowski S. Poly (1,1-lactide) microspheres by ring-opening polymerization. Macromolecules, 1996; 29:4556-64.

Sostaric T, Boyce MC, Spickett EE. Separation and identification of the organic acids in Angelicae Radix and Ligustici Rhizoma by HPLC and CE. Agric Food Chem, 2000; 48:5802-7.

Tamayo FG, Casillas JL, Martin-Esteban A. Evaluation of new selective molecularly imprinted polymers prepared by precipitation polymerisation for the extraction of phenylurea herbicides. J Chromatogr A, 2005; 1069:173-81.
Tavengwa NT, Cukrowska E, Chimuka L. Synthesis, adsorption and selectivity studies of N-propyl quaternized magnetic poly(4-vinylpyridine) for hexavalent chromium. Talanta, 2016; 116:670-7.

Vetrano AM, Heck DE, Mariano TM, Mishin V, Laskin DL, Laskin JD. Characterization of the oxidase activity in mammalian catalase. Biol Chem, 2005; 280:35372-81.

Yan H, Row KH. Characteristic and synthetic approach of molecularly imprinted polymer. Int J Mol Sci, 2006; 7:155-78.

Yilmaz E, RamstrHm O, Moller P, Sanchez D, Mosbach KJ. A facile method for preparing molecularly imprinted polymer spheres using spherical silica templates. Mater Chem, 2002; 12:1577.

Yu P, Sun QL, Li JF, Tan ZJ, Yan YS, Li CX. Magnetic imprinted nanomicrosphere attached to the surface of bacillus using Miniemulsion polymerization for selective recognition of 2,4,6-trichlorophenol from aqueous solutions. J Ind Eng Chem, 2015; 29:349-58.

\section{How to cite this article:}

Bhawani SA, Bakhtiar S, Roland R, Shafqat SR, Ibrahim M. Synthesis of molecularly imprinted polymers of vanillic acid and extraction of vanillic acid from spiked blood serum. J Appl Pharm Sci, 2020; 10(04):056-062. 